\title{
Dynamics and Energetics of Permeation Through Aquaporins. What Do We Learn from Molecular Dynamics Simulations?
}

\author{
Jochen S. Hub, Helmut Grubmüller, and Bert L. de Groot
}

\begin{abstract}
Contents
1 Why Molecular Dynamics Simulations? ....................... 58

2 Water Permeates Through AQPs Along a Lattice of Protein-Water Hydrogen Bonds ... . 58

$2.1 \quad$ Calculating Water Permeability Coefficients ...................... 61

2.2 Perfect Single-File Water Transport? . . . . . . . . . . . . . . . . . . . . . . 62

3 Protons Are Excluded by an Electrostatic Barrier ...................... 63

3.1 Origin of the Barrier: Protein Electric Field Vs. Desolvation Effects . . . . . . . . 65

4 Are Aquaporins Permeated by Gas? . . . . . . . . . . . . . . . . . . . . . . 65

5 Permeation of Uncharged Solutes Through Aquaporin Channels $\ldots \ldots \ldots \ldots \ldots \ldots \ldots 68$

5.1 Glycerol Permeation Through Aquaglyceroporin GlpF ............... 68

5.2 Toward a General Understanding of Channel Selectivity ................ 68

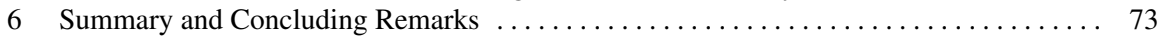

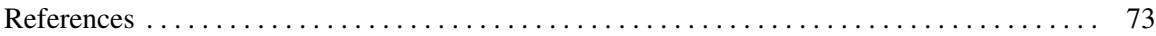

Abstract Aquaporins (AQPs) are a family of integral membrane proteins, which facilitate the rapid and yet highly selective flux of water and other small solutes across biological membranes. Molecular dynamics (MD) simulations contributed substantially to the understanding of the molecular mechanisms that underlie this remarkable efficiency and selectivity of aquaporin channels. This chapter reviews the current state of MD simulations of aquaporins and related aquaglyceroporins as well as the insights these simulations have provided. The mechanism of water permeation through AQPs and methods to determine channel permeabilities from simulations are described. Protons are strictly excluded from AQPs by a large electrostatic barrier and not by an interruption of the Grotthuss mechanism inside the pore. Both the protein's electric field and desolvation effects contribute to this barrier. Permeation of apolar gas molecules such as $\mathrm{CO}_{2}$ through AQPs is accompanied by a large energetic barrier and thus can only be expected in membranes with a low intrinsic gas permeability. Additionally, the insights from simulations into the mechanism of glycerol permeation through the glycerol facilitator GlpF from E. coli

B.L. de Groot ( $\varangle)$

Computational Biomolecular Dynamics Group, Max-Planck Institute for Biophysical Chemistry, Am Fassberg 11, 37077 Göttingen, Germany

bgroot@gwdg.de

E. Beitz (ed.), Aquaporins, Handbook of Experimental Pharmacology 190,

(C) Springer-Verlag Berlin Heidelberg 2009 
are summarized. Finally, MD simulations are discussed that revealed that the aromatic/arginine constriction region is generally the filter for uncharged solutes, and that AQP selectivity is controlled by a hydrophobic effect and steric restraints.

\section{Why Molecular Dynamics Simulations?}

Aquaporins (AQPs) facilitate water transport across biological membranes in response to an osmotic pressure (Preston et al. 1992). Compared with other biological processes, the translocation of water molecules by AQPs is extremely fast, on a nanosecond timescale (Zeidel et al. 1992). Remarkably, AQPs are also highly selective. Ions, in particular protons, are strictly excluded from AQPs, which ensures that electrochemical gradients across the membrane are maintained (Zeidel et al. 1994). Related aquaglyceroporins are permeated by larger solutes such as glycerol and/or urea whereas ordinary AQPs exclude any larger solutes. How can channels be efficient and highly selective at the same time?

High-resolution structures of AQPs have been determined by electron microscopy (Gonen et al. 2004; Murata et al. 2000) and X-ray diffraction experiments (Fu et al. 2000; Hiroaki et al. 2006; Lee et al. 2005; Savage et al. 2003; Sui et al. 2001; Törnroth-Horsefield et al. 2006). The structures provide invaluable insights in the molecular mechanisms acting in aquaporins. However, mostly static information was provided and we can therefore not observe aquaporins at work. So far, there is no experimental method of sufficient spatial and time resolution to monitor permeation through aquaporins on a molecular level. Molecular dynamics (MD) simulations therefore complement experiments by providing the progression of the biomlecular system at atomic resolution. Having all atomic coordinates as well as interaction energies and forces at hand, simulations yield insight into the physiochemical mechanisms (free energies, entropies, electrostatic forces, formation and rupture of hydrogen bonds, etc.), which drive biological processes such as permeation through aquaporins. The technique of MD simulations is sketched in Fig. 1.

During the last years, MD simulations of aquaporins have been a quite active field of research and provided (and still provide) remarkable insights into the function of these fascinating channels. This chapter overviews the current state of aquaporin simulations and shows how simulations reveal molecular mechanisms underlying the efficiency and selectivity of aquaporins, and thus explain biological function.

\section{Water Permeates Through AQPs Along a Lattice of Protein-Water Hydrogen Bonds}

High-resolution structures of aquaporin-1 (AQP1) (de Groot et al. 2001; Murata et al. 2000; Sui et al. 2001) and the bacterial glycerol facilitator GlpF (Fu et al. 2000) enabled atomistic real-time molecular dynamics (MD) simulations of spontaneous, 

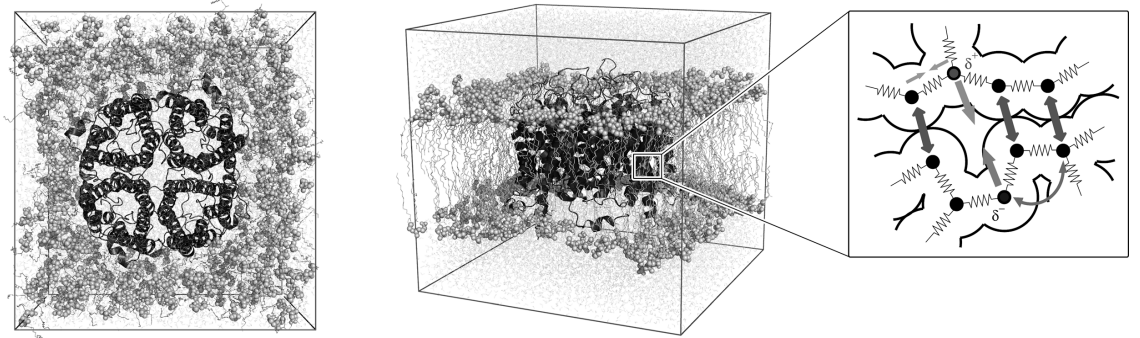

Fig. 1 Molecular dynamics simulations. Various kinds of interatomic forces act within macromolecules (here, an aquaporin tetramer). Forces arising from chemical bonds, here represented as springs, compel bound atoms into their equilibrium distances or equilibrium angles (thin arrows). Pauli repulsion (dark double arrows) prohibits atoms from penetrating through each other. Longrange interactions, particularly Coulomb interactions (thick light gray arrows) between partially charged atoms $\left(\delta^{+}, \delta^{-}\right)$, contribute significantly to the stability of a protein structure. All these interactions (and several others) determine the three-dimensional structure of a protein as well as the motion of each individual atom; they are therefore fully included within a molecular dynamics (MD) simulation. The movement of the atoms is calculated in classical approximation by numerical integration of Newton's equations of motion. This approximation holds at room temperature for many processes. Because the forces change rapidly with the changing atomic positions, all forces have to be repeatedly updated in small time steps (typically $10^{-15} \mathrm{~s}$ ). Thus, $10^{6}$ such integration steps simulate the movement of all atoms of the simulation system for the short time span of $1 \mathrm{~ns}$. To date, the typical length of MD simulations is $\approx 100 \mathrm{~ns}$, limited by the available computational resources.

full permeation events in aquaporins (de Groot and Grubmüller 2001; Tajkhorshid et al. 2002). It was found that both AQP1 and GlpF act as two-stage filters (de Groot and Grubmüller 2001). The first stage of the filter is located in the central part of the channel at the asparagine/proline/alanine (NPA) region; the second stage is located on the extracellular face of the channel in the aromatic/arginine ( $\mathrm{ar} / \mathrm{R})$ constriction region (cf. Fig. 2). An independent simulation of GlpF (Tajkhorshid et al. 2002) using a different force field confirmed the crucial role of the NPA region; this had also been inferred from the fact that this motif is highly conserved (Heymann and Engel 2000; Jung et al. 1994). These simulation studies also suggested mutants that change the permeation characteristics in a predicted manner (Tajkhorshid et al. 2002).

The simulations also addressed the energetics of water permeation. Overall, the channels achieve their high water permeability through a fine-tuned choreography of hydrogen bonds (de Groot and Grubmüller 2001). Whenever and wherever bulk water-water hydrogen bonds have to be ruptured to allow the water molecule to squeeze through the narrow NPA or ar/R regions, the protein offers replacement interactions, which largely compensate for the energetic cost of water-water bond rupture (cf. Fig. 3b). This remarkable complementarity to bulk water lowers the activation barrier to a large extent and thus allows the high permeation rate, which is observed both experimentally and in simulations, despite the hydrophobic nature of the pore. 


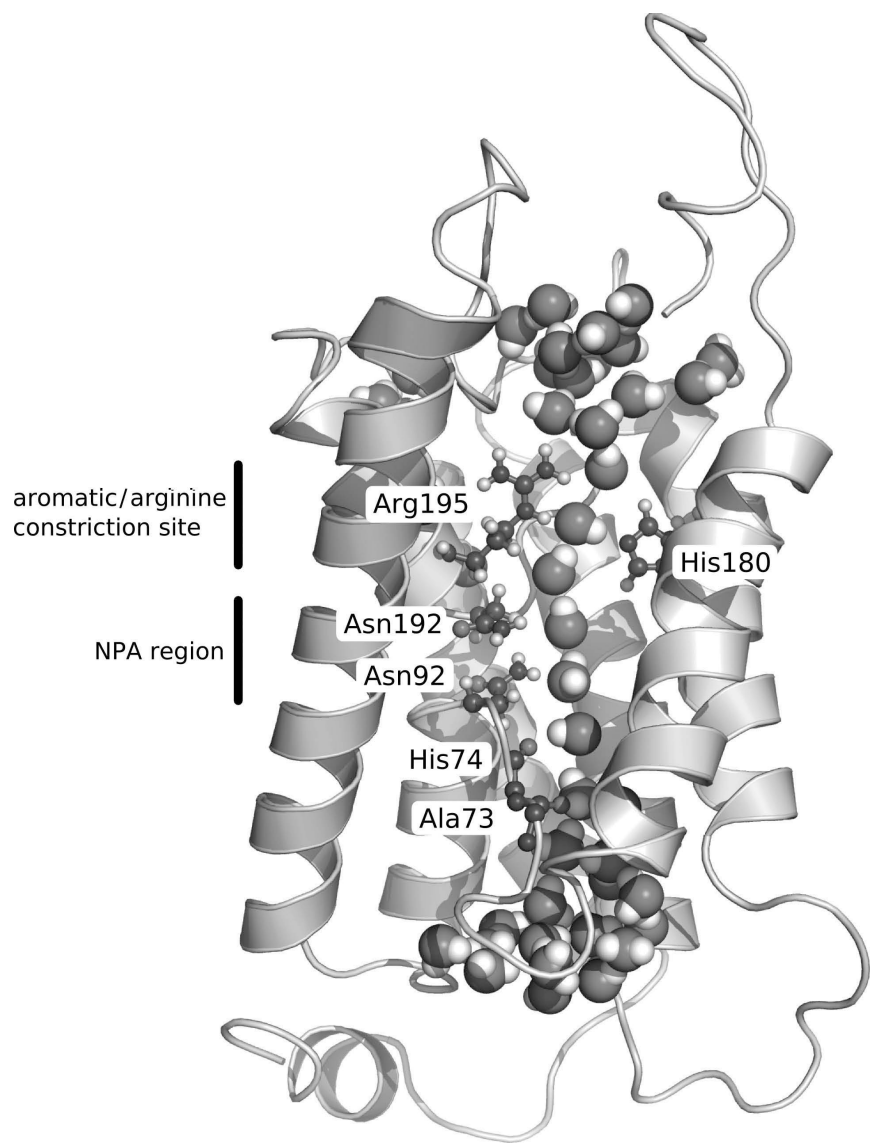

Fig. 2 Snapshot from an MD simulation of AQP1 showing a single file of water inside the AQP1 channel. Water molecules are shown as spheres, some water-interacting amino acid side chains are shown in ball-and-stick representation. As indicated by the black bar, the two conserved Asn-ProAla (NPA) motifs are located at the end of the two half helices HB and HE. The asparagines of the NPA motifs form strong hydrogen bonds to permeating water molecules. Closer to the extracellular exit of the channel, the aromatic/arginine region $(\mathrm{ar} / \mathrm{R})$ forms the narrowest part of the channel (de Groot et al. 2001; Sui et al. 2001).

The simulations finally revealed a pronounced water dipole orientation pattern across the channel, with the NPA region as its symmetry center (de Groot and Grubmüller 2001). In the simulations, the water molecules were found to rotate by $180^{\circ}$ on their path through the pore (Fig. 3a). By artificially switching off the electric dipoles of the half helices $\mathrm{B}$ and $\mathrm{E}$, it was elegantly demonstrated that it is the electrostatic field generated by the helical macrodipoles that mainly determines the strict water dipole orientation (Tajkhorshid et al. 2002). The dipolar rotation does not allow the water file to form a continuous hydrogen bond network inside the pore. 

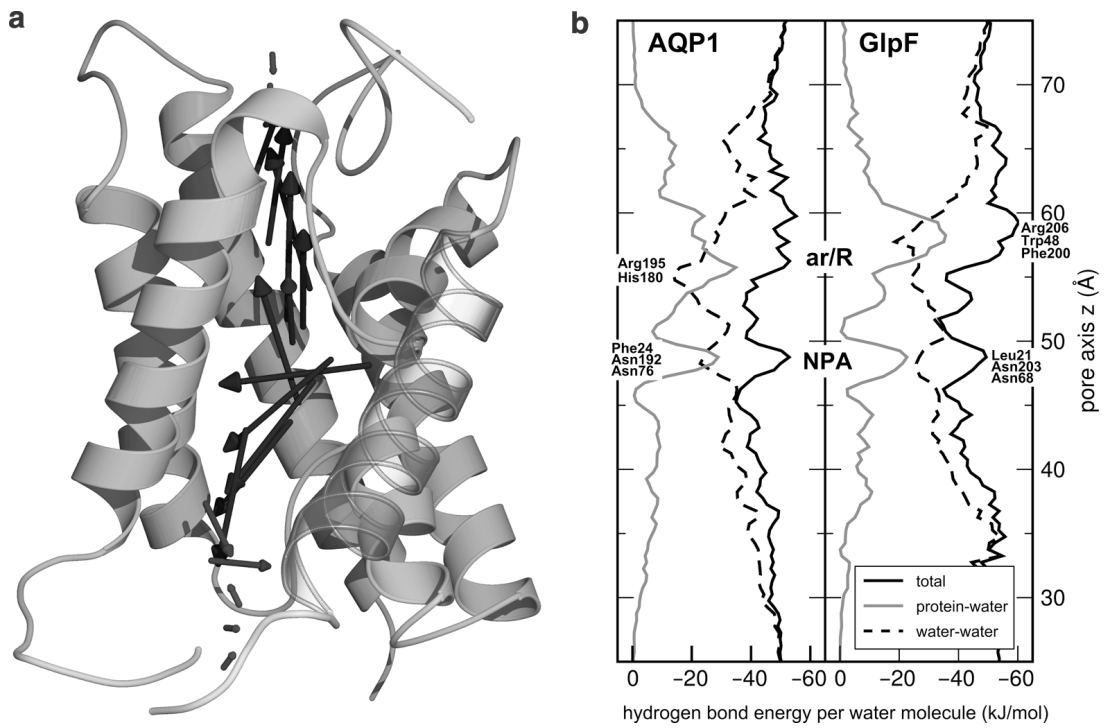

Fig. 3 (a) Bipolar orientation of water molecules inside the aquaporin-1 channel, as derived from MD simulations (de Groot and Grubmüller 2001). The water dipoles (black arrows) rotate by approximately $180^{\circ}$ while permeating though the AQP1 pore. (b) Hydrogen bond energies per water molecule (solid black lines) in AQP1 (left) and GlpF (right). Protein-water hydrogen bonds (gray) compensate for the loss of water-water hydrogen bonds (dashed). The main protein-water interaction sites are the ar/R region and the NPA site, apparent from the maxima in the (absolute) protein-water hydrogen bond energies (gray).

This fact led to speculations (Tajkhorshid et al. 2002) that it is the water orientation that prevents the channel from proton leakage (see also next section).

\subsection{Calculating Water Permeability Coefficients}

MD simulations allow one to address aquaporin function in quantitative terms. Calculation of permeability coefficients and comparison with measured values (Engel and Stahlberg 2002) provide a very sensitive test of the simulations. The best-studied permeability coefficient is the osmotic permeability $p_{\mathrm{f}}$. It can be defined from the net water flux $j_{w}$, which occurs in response to a difference in some solute concentration between the two water compartments $\Delta C_{\mathrm{s}}$. Then, $p_{\mathrm{f}}$ is given by (Finkelstein 1987)

$$
j_{w}=p_{\mathrm{f}} \Delta C_{s}
$$

The calculation of $p_{\mathrm{f}}$ from MD simulation is not straightforward. The reason is the following: under equilibrium conditions (without any osmotic pressure) a large number of water molecules cross the channel as result of thermal fluctuations. These frequent spontaneous channel crossings occur equally often in both directions of the channel, yielding zero net flux. After applying an osmotic pressure, the number of 
permeation events upward the chemical gradient is slightly reduced, yielding a net flux. The net flux is generally very small and is therefore difficult to detect against the large background of total channel crossings.

One strategy to overcome this problem is to apply a hydrostatic pressure instead of an osmotic pressure (Zhu et al. 2002). To generate a measurable net flux a very large pressure is however required, which, in turn, necessitates to artificially restrain the aquaporin in the simulation. Nevertheless, the obtained $p_{\mathrm{f}}$ is in good agreement to experiment.

An elegant alternative is to compute the nonequilibrium permeation coefficient $p_{\mathrm{f}}$ directly from equilibrium simulations. This approach is rigorous, because nonequilibrium quantities (such as transport coefficients) are closely related to equilibrium properties. Or more precisely, a thermodynamic system responds linearly to small external perturbations, and the response is quantitatively determined by equilibrium quantities of the system. This remarkable relation is referred to as fluctuation dissipation theorem. $p_{\mathrm{f}}$, for example, is related to spontaneous permeation events under equilibrium.

Spontaneous hops of a single file of water have for the first time been used to determine the $p_{\mathrm{f}}$ of gramicidin-A (de Groot et al. 2002). The method rests on the assumption that the permeation rate is proportional to a Boltzmann factor $\exp \left(-\Delta G^{\ddagger} / k_{B} T\right)$ with an Arrhenius activation energy $\Delta G^{\ddagger}$. (For an expanded derivation see $\mathrm{Zhu}$ et al. 2004b.) For AQP1, the method was reported to yield $p_{\mathrm{f}}=7.5 \times 10^{14} \mathrm{~cm}^{3} \mathrm{~s}^{-1}$ (de Groot and Grubmüller 2001, 2005) or $7.1 \times 10^{14} \mathrm{~cm}^{3} \mathrm{~s}^{-1}$ (Zhu et al. 2004b), in good agreement to experimental values of 3.2 to $11.7 \times$ $10^{14} \mathrm{~cm}^{3} \mathrm{~s}^{-1}$ (Engel and Stahlberg 2002). More recently, a model has been proposed, which describes the motions of all water molecules in the pore by one collective coordinate (Zhu et al. 2004a). The diffusion of this collective coordinate is again related to $p_{\mathrm{f}}$. The model has been successfully applied to a number of AQP channels, including mammalian AQP1 and AQP0 as well as the bacterial AQP-Z and GlpF (Hashido et al. 2005; Jensen and Mouritsen 2006). These studies found reasonable agreement to experimental $p_{\mathrm{f}}$ values for the water channels AQP1 and AQP-Z. The $p_{\mathrm{f}}$ of GlpF, however, was reported to be similar to the $p_{\mathrm{f}}$ of AQP-Z (Hashido et al. 2005), or even 2-3 times larger (Jensen and Mouritsen 2006), a finding which seems to contradict experiments (Borgnia and Agre 2001; Maurel et al. 1994). Further experiments and simulations are required to resolve this issue.

\subsection{Perfect Single-File Water Transport?}

The water permeation in AQPs is often referred to as single-file permeation. This picture may be supported by snapshots of MD simulations, which often display an ideal water file (compare Fig. 2). An idealized single-file structure requires, however, that no gaps between water molecules are present at any time, and that all water molecules in the channel move in a concerted fashion (Finkelstein 1987). In particular, water molecules must not interchange position. A number of MD stud- 
ies have investigated to which extent this ideal picture actually holds. One strategy is to compare the osmotic permeability $p_{\mathrm{f}}$ to the diffusive permeability coefficient $p_{\mathrm{d}}$. For a perfect single-file permeation $p_{\mathrm{f}} / p_{\mathrm{d}}=N+1$, where $N$ denotes the number of water binding sites inside the pore (or the average occupancy number if empty sites occur) (Finkelstein 1987). In AQP-Z $p_{\mathrm{f}} / p_{\mathrm{d}}$ was found to be $\approx 12$, close to the number of water molecules inside the pore, whereas $p_{\mathrm{f}} / p_{\mathrm{d}} \approx 4$ was found for GlpF (Jensen and Mouritsen 2006). Hence, the single-file structure is more pronounced in the narrow pore of AQP-Z.

Recently, Hashido et al. proposed a method to determine to which extent (1) concerted water motion and (2) uncorrelated local diffusion contribute to the total $p_{\mathrm{f}}$ (Hashido et al. 2007). The analysis revealed that water-water correlations are particularly reduced around the NPA region and that $p_{\mathrm{f}}$ is affected by slow local diffusion in the narrow ar/R region.

Taken together, such studies indicate that the picture of single-file permeation is an idealized simplification of the real situation and it only partly describes the permeation through AQPs. Long-range correlations between water molecules are reduced by water-protein interactions, and water molecules occasionally interchange positions, in particular in wider AQP channels such as GlpF.

\section{Protons Are Excluded by an Electrostatic Barrier}

Proton conduction in bulk water proceeds via the Grotthuss mechanism (de Grotthuss 1806). Accordingly, protons are transferred between water molecules via hydrogen bonds and transient hydronium ions, Eigen and Zundel clusters. Necessarily, the water dipoles reorient during this process. The observation of interrupted hydrogen bonds along the water chain inside the AQP pore (de Groot and Grubmüller 2001), as well as the strict orientation of the water molecules (de Groot and Grubmüller 2001; Tajkhorshid et al. 2002), led to speculation that these effects interfere with the Grotthuss mechanism and thus preclude proton conduction through the channel. Because these first-generation studies were mainly aimed at - and succeeded in explaining efficient water permeation, only (neutral) water molecules were considered and, hence, the aforementioned speculation about the mechanism of proton exclusion was based only on indirect evidence.

To obtain direct information, explicit treatments of excess protons and proton transfer reactions in second-generation simulations were required. Within only a few years, eight studies have been published, which explicitly address the energetics and dynamics of excess protons in the AQP channel (Burykin and Warshel 2003, 2004; Chakrabarti et al. 2004a, b; Chen et al. 2006; de Groot et al. 2003; Ilan et al. 2004; Kato et al. 2006). The applied methods are quite diverse, including classical electrostatics calculations (Chakrabarti et al. 2004b; de Groot et al. 2003; Jensen et al. 2003), Q-HOP proton transfer simulations (de Groot et al. 2003), semimicroscopic protein-dipole Langevin-dipole linear response approximation (PDLD/S-LRA) calculations (Burykin and Warshel 2003; de Groot et al. 2003; Kato et al. 2006), umbrella MD simulations employing the PM6 dissociable water model 
(Chakrabarti et al. 2004a, b), and steered (multistate) empirical valence bond proton transfer simulations (Chen et al. 2006; Ilan et al. 2004; Kato et al. 2006). Furthermore, the energetics of proton translocation have been computed for two different members of the aquaporin family, AQP1 and GlpF.

From these studies it became clear that the proton exclusion can not be explained from a discontinuous hydrogen bond network inside the channel, as inferred from the initial X-ray structures (Sui et al. 2001). Instead, if a proton is forced into the channel, remarkably high proton mobility through efficient Grotthuss transfers was observed throughout the channel, without any severe interruption (de Groot et al. 2003). These results contrast with the original picture of an interrupted proton wire. The water molecules inside the pore should in fact not be regarded as a static bipolar water column, with the water oxygen atoms pointed toward the channel center at any time. Instead, water molecules rotate inside the channel, and only the average water dipole is pointed toward the channel exits.

The consensus conclusion is that a large electrostatic barrier, rather than proton wire interruption effects, is the dominant mechanism of proton exclusion in aquaporins. From the free energy profile of proton translocation the barrier height was determined to approximately $25 \mathrm{kcal} \mathrm{mol}^{-1}$ (Chen et al. 2006; Kato et al. 2006), with the maximum of the profile being located in the NPA region (cf. Fig.4). Accordingly, the presence of a proton wire has little influence on a hypothetical proton transport, because the protons could not climb the barrier, even if the proton wire was intact.

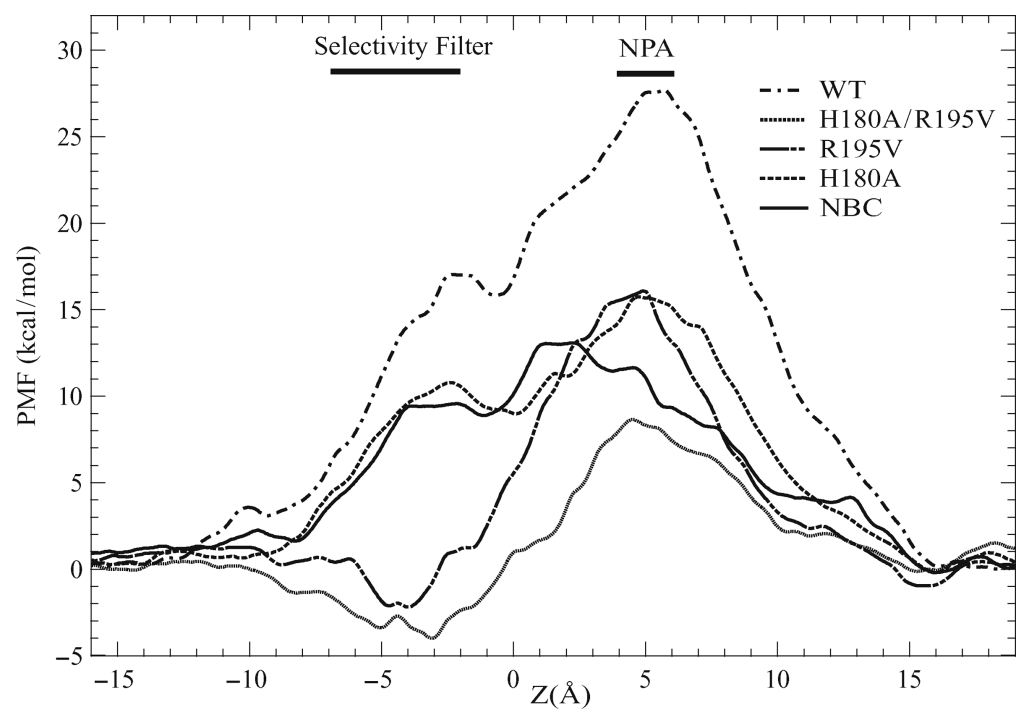

Fig. 4 Potentials of mean force (PMFs) for proton transfer through AQP1 (Chen et al. 2006). In the AQP1 wild type (WT, dotted-dashed curve), a large barrier of $28 \mathrm{kcal} \mathrm{mol}^{-1}$ prohibits any proton leakage. Switching off the dipoles of the half helices HB and HE (NBC, no-backbone-charge, solid curve) reduces the barrier substantially. Likewise, mutations in the aromatic/arginine region (here termed selectivity filter), such as R195V, H180A, R195V/H180A, reduce the barrier and may therefore cause proton leakage, as observed experimentally (Beitz et al. 2006). 


\subsection{Origin of the Barrier: Protein Electric Field Vs. Desolvation Effects}

A question that has been lively discussed and that is not yet completely resolved is the origin of the electrostatic barrier. Two competing pictures have been suggested. First, electric field generated by the dipoles of the two AQP half helices HB and HE has been proposed to repel the protons from the NPA region (Chakrabarti et al. 2004b; de Groot et al. 2003). This picture implies that the bipolar water orientation is only a side effect of the electrostatic field in the pore (de Groot et al. 2003), and not the cause of proton exclusion.

Others studies emphasized desolvation effects as the origin of the electrostatic barrier (Burykin and Warshel 2003; Kato et al. 2006). In the highly dielectric ( $\varepsilon=$ 80 ) bulk water, the proton is well solvated by surrounding water molecules. Upon moving the proton across the AQP pore, the solvation shell needs to be removed from the proton. Inside the pore, the hydronium ion is only partially solvated by few close water molecules, and the surrounding protein medium $[\varepsilon \approx 4$ (Kato et al. 2006)] stabilizes the hydronium only to a fraction of the solvation in the bulk. Thus, a large energetic cost results for moving the hydronium from the water into the channel.

The controversy on the origin of the electrostatic barrier is mainly caused by the problem of how to measure the contributions of polar groups (mainly of the half helices) to the barrier. A common approach is to switch off the corresponding partial charges during the simulation (Chakrabarti et al. 2004a; Chen et al. 2006). After such an alchemical transformation, the protein atoms rearrange toward a new stable configuration. The protein may even become unstable, and artificial restraints may be required to keep the protein in its native structure. To which degree the rearrangement of protein atoms should be allowed by the simulation protocol seems somewhat unclear, but has an impact on the quantitative results (Kato et al. 2006). The most recent results indicate that $35-55 \%$ of the free energy barrier is generated by the dipoles of the half helices HB and HE (Chen et al. 2006; Kato et al. 2006), and the remaining part is caused by desolvation (compare Fig. 4).

\section{Are Aquaporins Permeated by Gas?}

It has been a long-standing question whether aquaporins also facilitate gas permeation. In particular, the role of AQP1 as a $\mathrm{CO}_{2}$ channel has been a matter of lively debate ever since it has been reported that AQP1 increases the $\mathrm{CO}_{2}$ permeability of Xenopus oocytes membranes (Nakhoul et al. 1998). By now, aquaporins have been reported to increase the $\mathrm{CO}_{2}$ permeability of membranes of oocytes (Cooper and Boron 1998; Nakhoul et al. 1998), liposomes (Prasad et al. 1998), and red blood cells (Blank and Ehmke 2003; Endeward et al. 2006). Other studies did not report any impact of AQP1 on membrane $\mathrm{CO}_{2}$ permeability (Yang et al. 2000). Moreover, a physiological role of AQP1 or AQP5 for $\mathrm{CO}_{2}$ transport in the lung or 
in the kidney has been questioned (Fang et al. 2002). Another process for which aquaporin-mediated $\mathrm{CO}_{2}$ permeation has been suggested to play a physiological role is photosynthesis. It was shown that the leaf growth and the diffusion of $\mathrm{CO}_{2}$ inside the leaves of tobacco plants were dependent on the level of NtAQP1 expression, an aquaporin homologous to human AQP1 (Uehlein et al. 2003). By now, this question is still not settled by experiments.

This controversy triggered MD simulations of $\mathrm{CO}_{2}$ permeation through AQP1 (Hub and de Groot 2006). Potentials of mean force (PMFs) were computed of possible pathways for $\mathrm{CO}_{2}$ across an AQP1 tetramer, embedded in a model membrane of pure POPE (1-palmitoyl-2-oleoyl-sn-glycero-3-phosphoethanolamine) (Fig. 5). The key finding was that $\mathrm{CO}_{2}$ encounters a substantial barrier of approximately $23^{\circ} \mathrm{kJ} \mathrm{mol}^{-1}$ when permeating through the AQP1 water pore (Fig. 5, solid curve). The corresponding PMF for the central cavity of the AQP tetramer displays two barriers of only $13^{\circ} \mathrm{kJ} \mathrm{mol}^{-1}$, assuming that the cavity is not blocked by an ion or organic molecule. Hence, the central cavity is more likely to contribute to a $\mathrm{CO}_{2}$ flux than the AQP1 water pores. A model POPE membrane, in contrast, was found to be highly permeable to $\mathrm{CO}_{2}$ with barriers of only $4 \mathrm{~kJ} \mathrm{~mol}^{-1}$ and a large membrane permeability of $P_{\mathrm{f}} \approx 12 \mathrm{~cm} \mathrm{~s}^{-1}$. AQP1 embedded in a membrane of POPE is therefore not expected to increase the $\mathrm{CO}_{2}$ permeation. Therefore, AQP1 can be expected to play a physiological role only in membranes with a low intrinsic $\mathrm{CO}_{2}$ permeability. Membranes with similar physicochemical characteristics to POPE are highly permeable to $\mathrm{CO}_{2}$, rendering a physiological role for AQP1mediated $\mathrm{CO}_{2}$ permeation in such membranes unlikely.

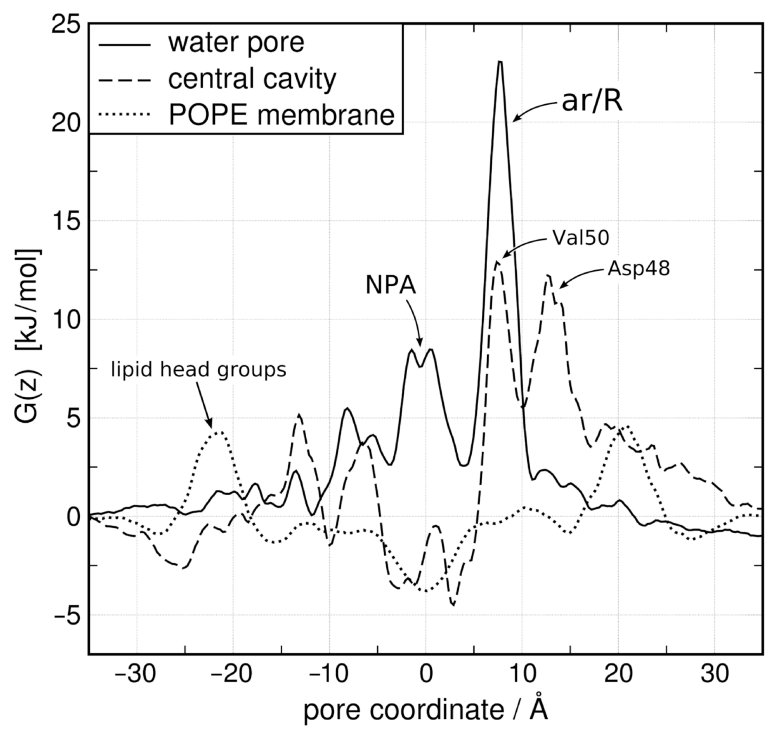

Fig. 5 Potentials of mean force $G(z)$ for $\mathrm{CO}_{2}$ permeation along possible pathways across an AQP1 tetramer embedded in a bilayer of POPE: through the AQP1 water pore (black solid line), the tetrameric central cavity (dashed), and across the POPE lipid bilayer (dotted). 
More recently, Wang et al. corroborated this picture (Wang et al. 2007) and showed that it holds qualitatively also to molecular oxygen. Like $\mathrm{CO}_{2}$, also $\mathrm{O}_{2}$ might permeate through the apolar central cavity. Interestingly, a small apolar cavity located between neighboring AQP1 monomers was suggested to be permeable to $\mathrm{O}_{2}$. Compared with a membrane of POPE, however, the AQP1 pores are not expected to contribute substantially to $\mathrm{O}_{2}$ permeation.

From the MD simulations it was possible to extract the molecular mechanism underlying the $23-\mathrm{kJ} \mathrm{mol}^{-1}$ barrier for $\mathrm{CO}_{2}$ permeation through the AQP1 water pore (Hub and de Groot 2006). The main barrier is located in the ar/R region (cf. Fig. 5). The barrier has been proposed to originate from water-protein hydrogen bonds, mainly to Arg195. If no $\mathrm{CO}_{2}$ molecule is present in the ar/R region, water forms hydrogen bonds to the guanidinium group of Arg195 (Fig. 6a, compare also Fig. 3b). Upon permeation of $\mathrm{CO}_{2}$ through the narrow ar/R site these water-Arg 195 hydrogen bonds are partially lost, leaving an energetically unfavorable configuration (Fig. 6b). Hence, since the ar/R site of AQP1 is both narrow and hydrophilic it generates a substantial barrier against permeation of the apolar $\mathrm{CO}_{2}$. This observation implies that the wider and more hydrophobic ar/R site of aquaglyceroporins is expected to be more permeable to $\mathrm{CO}_{2}$. Indeed, the main barrier for $\mathrm{CO}_{2}$ permeation through the bacterial aquaglyceroporin GlpF was recently determined to be only $13.5 \mathrm{~kJ} \mathrm{~mol}^{-1}$ (Hub and de Groot 2008), close to the barrier for water permeation.
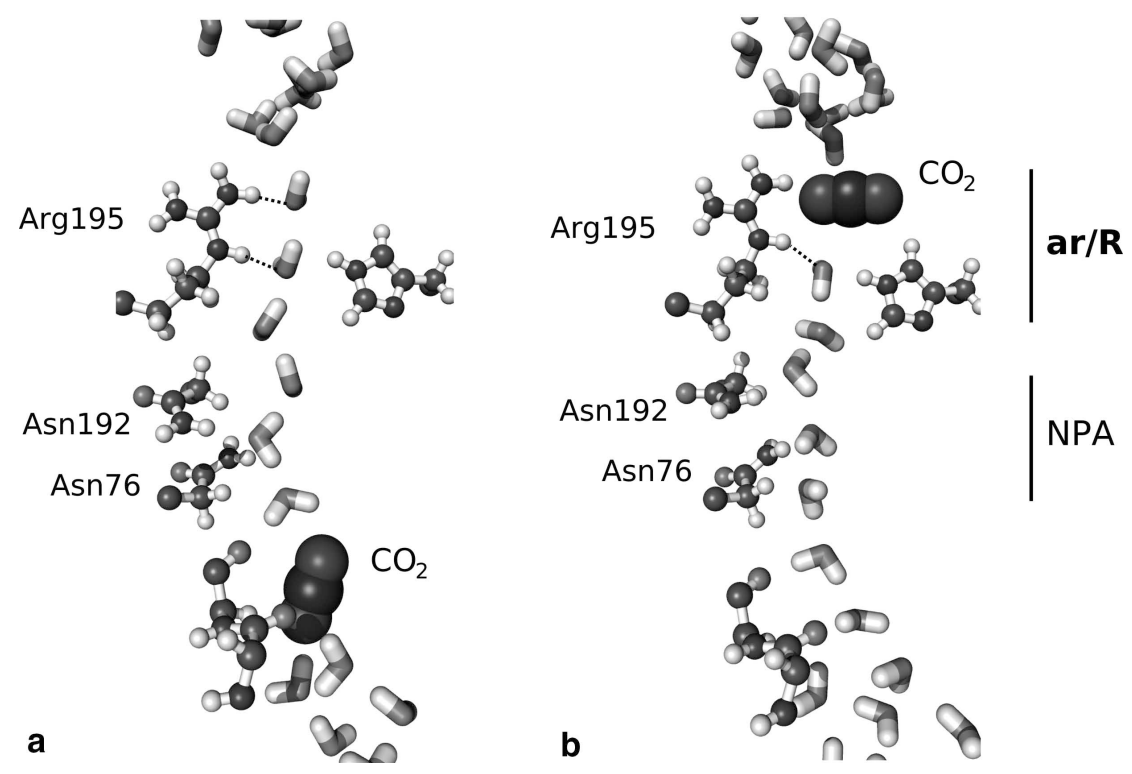

Fig. 6 Snapshots from a putative permeation event of a $\mathrm{CO}_{2}$ molecule through the AQP1 water pore, as derived from MD simulations. Water molecules are shown as sticks, the $\mathrm{CO}_{2}$ as dark spheres. (a) Water forms strong hydrogen bonds to the conserved arginine, as indicated by dotted lines. (b) Upon $\mathrm{CO}_{2}$ permeation through the ar/R region, such water-Arg 195 hydrogen bonds are (partially) lost, generating a substantial barrier against $\mathrm{CO}_{2}$ permeation (cf. also Fig. 5). 


\section{Permeation of Uncharged Solutes Through Aquaporin Channels}

\subsection{Glycerol Permeation Through Aquaglyceroporin GlpF}

The 2.2- $\AA$-resolution structure of E. coli glycerol facilitator GlpF (Fu et al. 2000) opened the possibility to study the mechanism as well as the energetics of glycerol permeation through GlpF. The simulations revealed that glycerol is conducted via repeated formation and rupture of glycerol-protein hydrogen bonds (Jensen et al. 2001). Water is actively involved in this process as it competes with glycerol for hydrogen bonds to the protein. Using Jarzynski's equality, the potential of mean force (PMF) for glycerol permeation through GlpF was reconstructed from nonequilibrium simulations (Jensen et al. 2002). The PMF displayed a periplasmic vestibule of low energy, which was speculated to enhance the uptake of glycerol from the environment. The existence of this vestibule could however not be confirmed by more recent MD studies (Hénin et al. 2008; Hub and de Groot 2008).

Permeability measurements of chiral polyols have suggested that GlpF is stereoselective (Heller et al. 1980). Interestingly, the stereoselectivity has also been observed in MD simulations. The force that is required to pull glycerol through the $\mathrm{ar} / \mathrm{R}$ region of GlpF depends on the orientation of the glycerol molecule inside the pore (Jensen et al. 2002). This effect is caused by the arrangement of potential hydrogen bond partners inside the channel: in the favorable orientation in the ar/R site, all three hydroxyl groups of glycerol are able to form hydrogen bonds to polar protein groups or to the nearby water molecules. In the unfavorable orientation, however, one hydroxyl group gets in close contact to the apolar side chain of Phe200, which accounts for an unfavorable configuration. Recently it has been suggested that different conformations of the two $\mathrm{O}-\mathrm{C}-\mathrm{C}-\mathrm{O}$ torsional angles play a role in glycerol conduction through GlpF (Hénin et al. 2008). In these simulations, the probabilities for the two torsional angles to be in the gauche or anti state highly depend on the glycerol position along the channel. Hence, internal transitions of the glycerol molecule may be required for permeation.

\subsection{Toward a General Understanding of Channel Selectivity}

So far, 13 different AQP channels were identified in humans (Zardoya 2005). All AQPs are assumed to share a common fold and a number of conserved residues in the channel, such as the conserved NPA motifs and the arginine in the ar/R site (Heymann and Engel 2000). In spite of these remarkable similarities, 13 distinct AQPs have evolved, reflecting the need for tight control of membrane permeability for water and other solutes.

Fine-tuned differences between AQP family members in channel diameter and the arrangement of hydrogen bond partners may determine their permeabilities with respect to different solutes. However, the molecular mechanisms that determine the 
AQPs' substrate specificities were poorly understood until recently. Therefore, a recent MD study investigated the selectivity of one member of each of the two AQP subfamilies, i.e., AQP1 as a representative for the AQP water channels, and E. coli GlpF as a member of the aquaglyceroporin family (Hub and de Groot 2008). Umbrella sampling simulations (Torrie and Valleau 1974) were employed to compute PMFs for the permeation of a number of solutes through AQP1 and GlpF (Fig. 7).

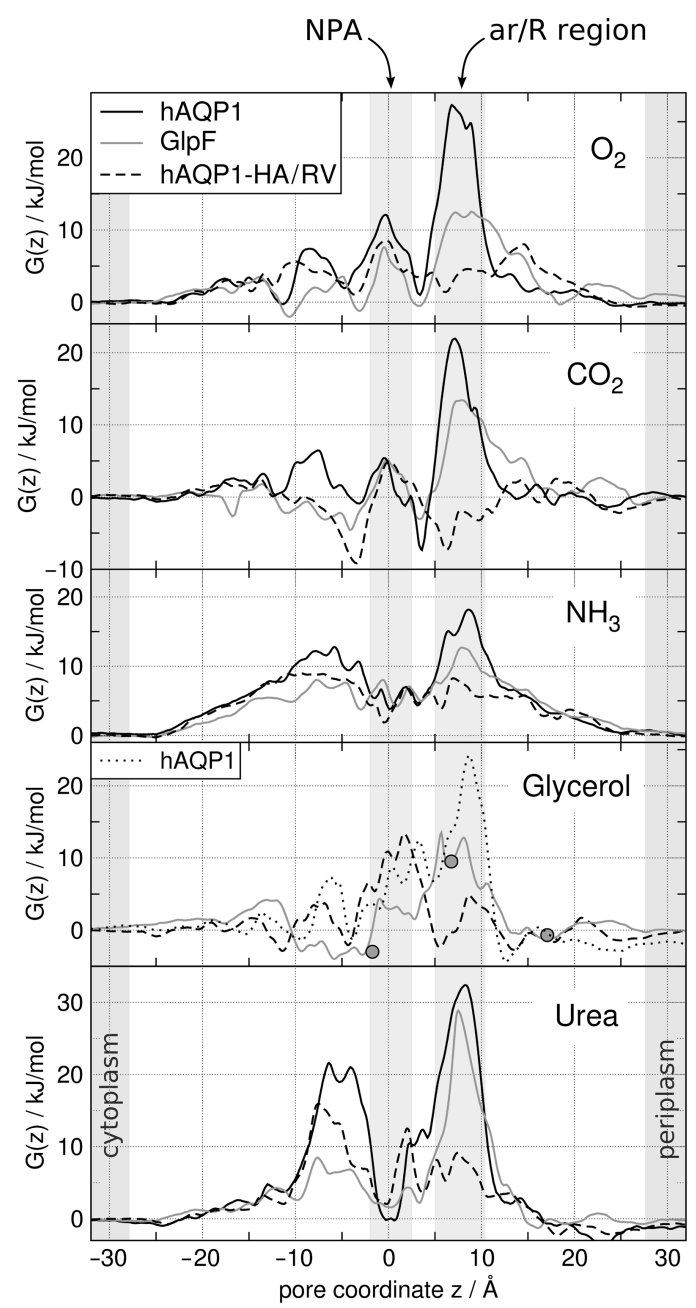

Fig. 7 Potentials of mean force $G(z)$ for the permeation of $\mathrm{O}_{2}, \mathrm{CO}_{2}, \mathrm{NH}_{3}$, glycerol, and urea through AQP1 (black solid curves) and GlpF (gray). The NPA site and the ar/R region are highlighted by gray bars. Note that the main barriers are located in the ar/R region, demonstrating its role for the selectivity of AQPs for uncharged solutes. The glycerol positions in the GlpF crystal structure are shown as small circles (Fu et al. 2000). The barrier against glycerol permeation through AQP1 (dotted line) may have been underestimated in the simulation protocol, see Hub and de Groot (2008) for details. Mutations in the ar/R region (dashed lines) have drastic effects on the main barrier and therefore on channel selectivity. 
The considered solutes included $\mathrm{O}_{2}, \mathrm{CO}_{2}, \mathrm{NH}_{3}, \mathrm{H}_{2} \mathrm{O}$, glycerol, and urea, which differ substantially in hydrophobicity and size. All computed PMFs display a main barrier in the ar/R region, confirming its role as the selectivity filter for uncharged solutes. GlpF was found to be generally less selective than AQP1.

To address whether a solute is likely to permeate through a channel, the permeability of the channel must be compared with the permeability of the surrounding lipid bilayer. In terms of energetic barriers, permeation through the channel is only expected if the barrier for channel permeation is substantially lower than the barrier for permeation across the lipid bilayer. Therefore, PMFs for solute permeation through two model membranes, composed of pure POPC and pure POPE, respectively, have been computed from simulations (Hub and de Groot 2008). The PMFs for POPC are displayed in Fig. 8.

By comparing the AQP PMFs (Fig. 7) with the membrane PMFs (Fig. 8) a number of conclusions can be drawn. For example, membranes similar to POPE or POPC are rapidly permeated by apolar gas molecules such as $\mathrm{O}_{2}$ and $\mathrm{CO}_{2}$. Neither AQP1 nor GlpF are expected to enhance the flux of $\mathrm{O}_{2}$ or $\mathrm{CO}_{2}$ in such membranes (compare previous section). AQP1 and GlpF could potentially be permeated by ammonia. However, only the barrier for $\mathrm{NH}_{3}$ permeation through $\mathrm{GlpF}$ is substantially lower than the membrane barriers. Therefore, $\mathrm{GlpF}$ is expected to enhance $\mathrm{NH}_{3}$ flux while AQP1 is not, in line with experimental findings (Holm et al. 2005).

It is illustrative to display permeation barriers as a function of the hydrophobicity of the permeating solute (Fig. 9). The plot demonstrates that the barrier height for the permeation of small solutes through AQP1 correlates with solute hydrophobicity (Fig. 9a). Larger solutes such as glycerol and urea are sterically excluded from

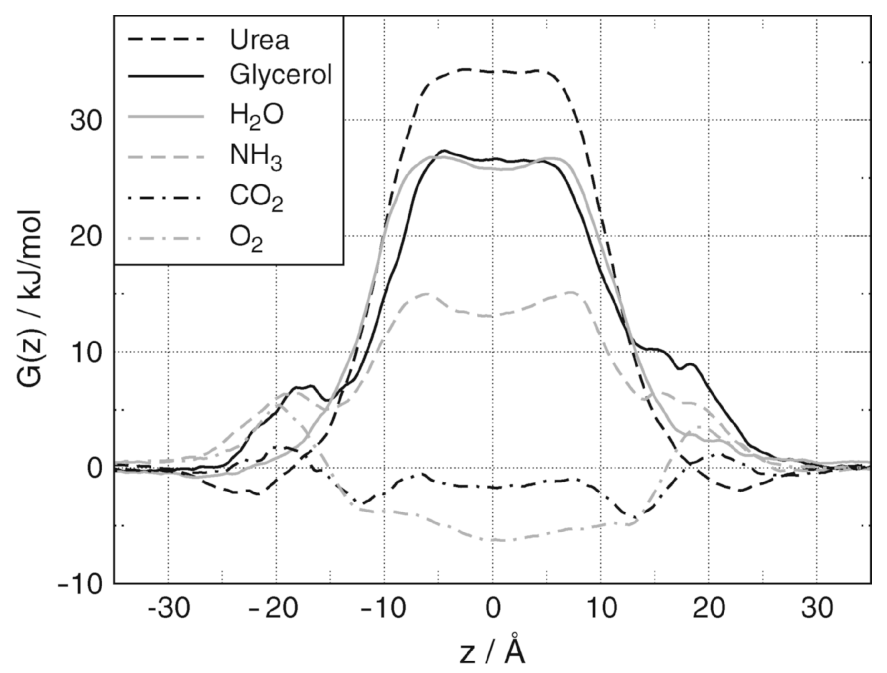

Fig. 8 Potentials of mean force for solute permeation through a membrane of POPC, as indicated in the legend. The membrane is highly permeable to apolar gas molecules such as $\mathrm{O}_{2}$ and $\mathrm{CO}_{2}$, whereas urea, glycerol, and water require a channel for a rapid flux across the membrane. 


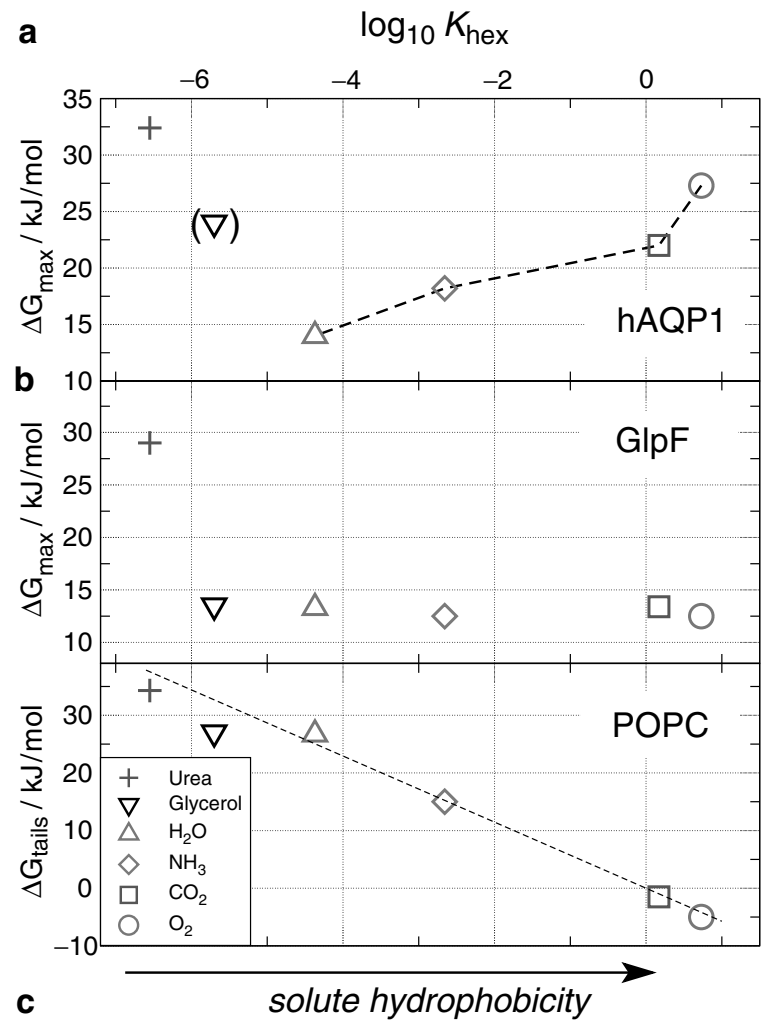

Fig. 9 Permeability as a function of solute hydrophobicity. The hexadecane-water partition coefficient $K_{\text {hex }}$ is used as a measure for hydrophobicity. The main barrier height $\Delta G_{\max }$ for solute permeation through AQP1 (a) and GlpF (b) vs. $\log _{10} K_{\text {hex }}$. AQP1 forms a filter against both hydrophobicity and size, whereas GlpF is permeable to all considered solutes except for urea (compare legend). (c) Solvation free-energy difference $\Delta G_{\text {tails }}$ between the solute in the bulk water and in the hydrophobic environment between the lipid tails. The measured energetic cost for moving the solute from water into hexadecane is shown for comparison as a dotted line.

AQP1. Taken together, the ar/R region of AQP1 can be considered as a filter that allows the permeation of small polar solutes. Note that this filter mechanism does not apply in GlpF (Fig. 9b). Its larger and more hydrophobic ar/R region allows the rapid permeation of all considered solutes except for urea.

As can be seen from Fig. 9, the permeation characteristics of AQP1 and GlpF are quite different. MD simulations revealed two molecular mechanisms underlying the different selectivities of AQP water channels and aquaglyceroporins. First, larger solutes such as glycerol are sterically excluded from narrow water pores such as AQP1 or E. coli AQP-Z (Hub and de Groot 2008; Wang et al. 2005), a finding that has already been suggested from the pore size of the crystal structures $(\mathrm{Fu}$ et al. 2000; Savage et al. 2003; Sui et al. 2001). Second, given a solute fits sterically through the pore, water-protein interactions play an important role in channel selectivity, in particular inside the ar/R region. When a small solute permeates 
a human AQP1 GIpF

b
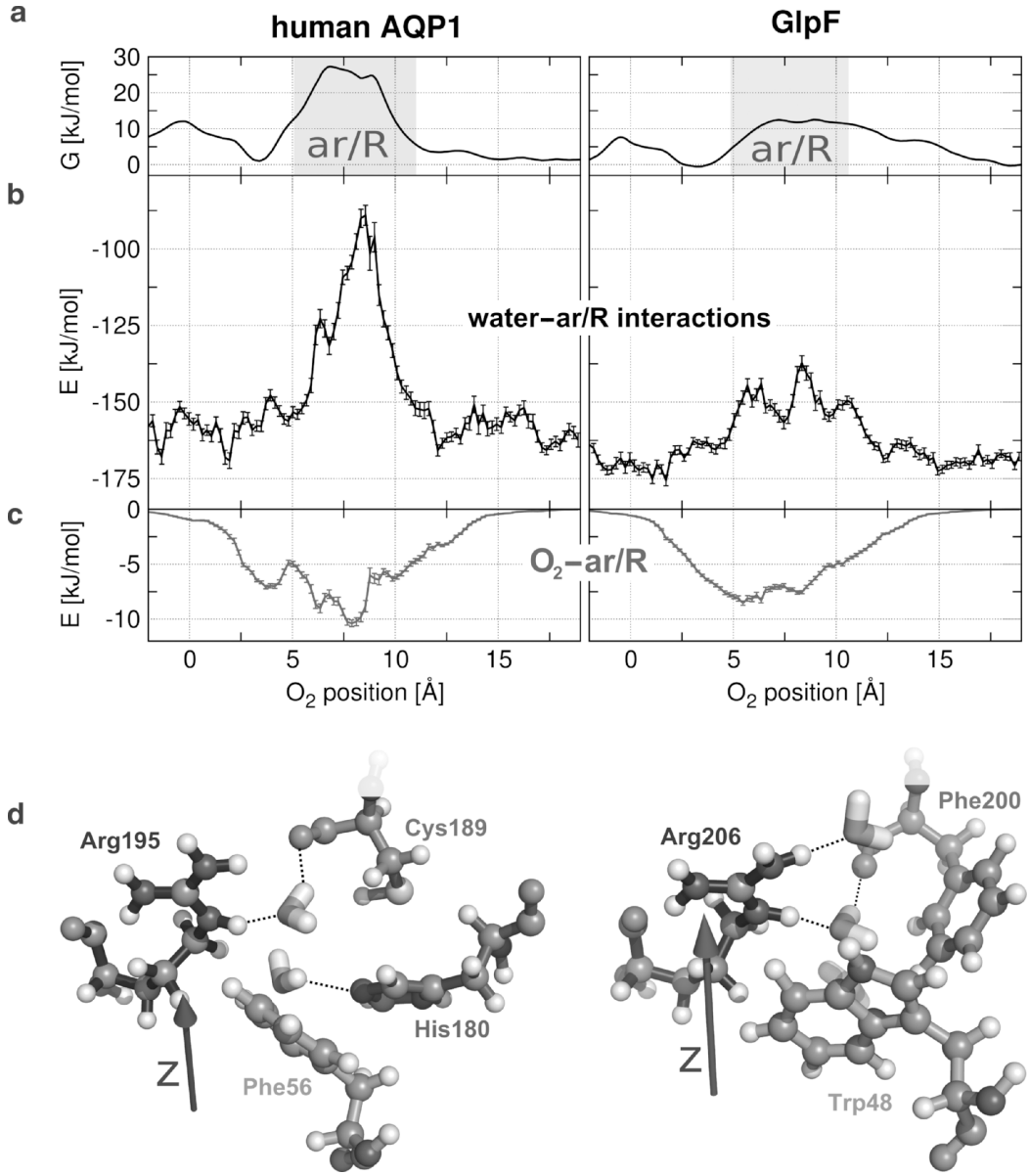

Fig. 10 Water-protein interactions as selectivity filter for aquaporins: analysis of interaction energies during a permeation event of $\mathrm{O}_{2}$ through AQP1 (left) and GlpF (right). (a) PMFs for $\mathrm{O}_{2}$ permeation through the $\mathrm{ar} / \mathrm{R}$ regions of $\mathrm{AQP} 1$ and $\mathrm{GlpF}$. (b) Interaction energies between water and the $\operatorname{ar} / \mathrm{R}$ residues as a function of $\mathrm{O}_{2}$ position. Water-protein interactions are reduced by $\approx 60 \mathrm{~kJ} \mathrm{~mol}^{-1}$ during $\mathrm{O}_{2}$ permeation through AQP1. (c) $\mathrm{O}_{2}$-protein interactions are weak $\left(\sim 10 \mathrm{~kJ} \mathrm{~mol}^{-1}\right)$ and cannot compensate for the loss of water-protein interaction. When $\mathrm{O}_{2}$ permeates through $\mathrm{GlpF}(\mathbf{b}$, right), water-protein interactions are much less reduced than in AQP1, rendering a lower barrier for $\mathrm{O}_{2}$ permeation through GlpF compared with AQP1. (d) Snapshots from MD simulations showing the ar/R regions of AQP1 and GlpF. Possible water-protein hydrogen bonds are indicated by dashed lines.

through the narrow and hydrophilic ar/R site of AQP1, water-protein interactions (mainly to the conserved arginine) are substantially reduced (cf. Fig. 10b, left) and replaced by solute-protein interactions (Fig. 10c). More hydrophilic the solutes interact more strongly with the polar groups in the ar/R region implying a lower cost to replace the water molecule. This hydrophobic effect leads to the correlation between solute hydrophobicity and barrier height in AQP1. The ar/R region of 
aquaglyceroporins such as GlpF is wider and more hydrophobic (Fu et al. 2000). Therefore, water-protein interactions are hardly reduced upon permeation of small solutes through GlpF (Fig. 10b, right), rendering GlpF an efficient channel for small uncharged solutes. Taken together, water-pore interactions complemented by steric restraints emerge as the determinants underlying channel selectivity.

\section{Summary and Concluding Remarks}

Providing atomic coordinates and interaction energies at high time and special resolution, MD simulations have proved to be a powerful tool to investigate molecular mechanisms of solute and water permeation through AQP channels. Possible limitations of the applied force field and the simulation protocol as well as the need for comparison to experimental data should however be kept in mind. For many quantities, such as permeability coefficients, the agreement between simulation and experiment is favorable, providing a direct means of cross validation. Occasional but striking discrepancies - such as on the water permeability of GlpF - are expected to trigger further simulations or experiments that eventually resolve such issues.

Within only a few years, simulations enabled us to obtain a quite detailed understanding of aquaporin function. Water flux through AQPs may be roughly considered as single-file permeation, although interruptions of the single-file structure are quite frequent. The ordered water structure in the channel is ensured by the frequent arrangement of hydrogen bond partners along the pore, which also compensate for the loss of solvation when water molecules enter the channel. The dipoles of two half helices HB and HE generate an electrostatic field inside the pore, which make the water dipoles rotate by $180^{\circ}$ upon permeation. Protons are excluded from AQPs by a large electrostatic barrier, which has its maximum at the NPA site. The contributions of polar protein groups and of desolvation effects to the barrier are still a matter of debate.

The aromatic/arginine ( $\mathrm{ar} / \mathrm{R})$ region is the selectivity filter for uncharged solutes. Small solutes are filtered through a hydrophobic effect. Whether and under which conditions AQPs facilitate gas, remains an intriguing question. For larger solutes such as glycerol, steric restraints combined with the arrangement of hydrogen bond donors and acceptors determine channel selectivity.

Acknowledgment This work was supported by EU grant no. LSHP-CT-2004-012189.

\section{References}

Beitz E, Wu B, Holm LM, Schultz JE, Zeuthen T (2006) Point mutations in the aromatic/arginine region in aquaporin 1 allow passage of urea, glycerol, ammonia, and protons. Proc Natl Acad Sci USA 103:269-274

Blank ME, Ehmke $\mathrm{H}$ (2003) Aquaporin-1 and $\mathrm{HCO}_{3}(-)-\mathrm{Cl}(-)$ transporter-mediated transport of $\mathrm{CO}_{2}$ across the human erythrocyte membrane. J Physiol 550:2:419-429 
Borgnia MJ, Agre P (2001) Recontitution and functional comparison of purified GlpF and AqpZ, the glycerol and water channels from Eschericia coli. Proc Natl Acad Sci USA 98:2888-2893

Burykin A, Warshel A (2003) What really prevents proton transport through aquaporin? Charge self-energy versus proton wire proposals. Biophys1 J 85:3696-3706

Burykin A, Warshel A (2004) On the origin of the electrostatic barrier for proton transport in aquaporin. FEBS Lett 570:41-46

Chakrabarti N, Roux B, Pomes R (2004a) Structural determinants of proton blockage in aquaporins. J Mol Biol 343:493-510

Chakrabarti N, Tajkhorshid E, Roux B, Pomes R (2004b) Molecular basis of proton blockage in aquaporins. Structure 12:65-74

Chen $\mathrm{H}$, Wu Y, Voth GA (2006) Origins of proton transport behavior from selectivity domain mutations of the aquaporin-1 channel. Biophys J 90:L73-L75

Cooper GJ, Boron WF (1998) Effect of PCMBS on $\mathrm{CO}_{2}$ permeability of Xenopus oocytes expressing aquaporin 1 or its C189S mutant. Am J Physiol 275:C1481-C1486

de Groot BL, Grubmüller H (2001) Water permeation across biological membranes:mechanism and dynamics of aquaporin-1 and GlpF. Science 294:2353-2357

de Groot BL, Grubmüller H (2005) The dynamics and energetics of water permeation and proton exclusion in aquaporins. Curr Opin Struct Biol 15:176-183

de Groot BL, Engel A, Grubmüller H (2001) A refined structure of human Aquaporin-1. FEBS Lett 504:206-211

de Groot BL, Tieleman DP, Pohl P, Grubmüller H (2002) Water permeation through gramicidin A:desformylation and the double helix; a molecular dynamics study. Biophys J. 82:2934-2942

de Groot BL, Frigato T, Helms V, Grubmüller H (2003) The mechanism of proton exclusion in the aquaporin-1 water channel. J Mol Biol 333:279-293

de Grotthuss CJT (1806) Sur la décomposition de l'eau et des corps qu'elle tient en dissolution à l'aide de l'électricité galvanique. Ann Chim LVIII:54-74

Endeward V, Musa-Aziz R, Cooper GJ, Chen L-M, Pelletier MF, Virkki LV, Supuran CT, King LS, Boron WF, Gros G (2006) Evidence that aquaporin 1 is a major pathway for $\mathrm{CO}_{2}$ transport across the human erythrocyte membrane. FASEB J 20:1974-1981

Engel A, Stahlberg H (2002) Aquaglyceroporins: channel proteins with a conserved core, multiple functions and variable surfaces. Int. Rev. Cytol.215:75-104

Fang X, Yang B, Matthay MA, Verkman AS (2002) Evidence against aquaporin-1-dependent $\mathrm{CO}_{2}$ permeability in lung and kidney. J Physiol 542:63-69

Finkelstein A (1987) Water movement through lipid bilayers, pores, and plasma membranes. Wiley, New York

Fu D, Libson A, Miercke LJ, Weitzman C, Nollert P, Krucinski J, Stroud RM (2000) Structure of a glycerol-conducting channel and the basis for its selectivity. Science 290:481-486

Gonen T, Sliz P, Kistler J, Cheng Y, Walz T (2004) Aquaporin-0 membrane junctions reveal the structure of a closed water pore. Nature 429:193-197

Hashido M, Ikeguchi M, Kidera A (2005) Comparative simulations of aquaporin family: AQP1, AQPZ, AQP0 and GlpF. FEBS Lett 579:5549-5552

Hashido M, Kidera A, Ikeguchi M (2007) Water transport in aquaporins: osmotic permeability matrix analysis of molecular dynamics simulations. Biophys J 93:373-385

Heller KB, Lin EC, Wilson TH (1980) Substrate-specificity and transport-properties of the glycerol facilitator of Eschericia coli. J Bacteriol 144:274-278

Hénin J, Tajkhorshid E, Schulten K, Chipot C (2008) Diffusion of glycerol through Escherichia coli aquaglyceroporin GlpF. Biophys J 94:832-839

Heymann JB, Engel A (2000) Structural clues in the sequences of the aquaporins. J Mol Biol 295:1039-1053

Hiroaki Y, Tani K, Kamegawa A, Gyobu N, Nishikawa K, Suzuki H, Walz T, Sasaki S, Mitsuoka K, Kimura K, Mizoguchi A, Fujiyoshi Y (2006) Implications of the aquaporin-4 structure on array formation and cell adhesion. J Mol Biol 355:625-639

Holm LM, Jahn TP, Møller ALB, Schjoerring JK, Ferri D, Klaerke DA, Zeuthen T (2005) $\mathrm{NH}_{3}$ and $\mathrm{NH}_{4}{ }^{+}$permeability in aquaporin-expressing Xenopus oocytes. Pflugers Arch 450:415-428 
Hub JS, de Groot BL (2006) Does $\mathrm{CO}_{2}$ permeate through Aquaporin-1? Biophys J 91:842-848

Hub JS, de Groot BL (2008) Mechanism of selectivity in aquaporins and aquaglyceroporins. Proc Natl Acad Sci USA 105:1198-1203

Ilan B, Tajkhorshid E, Schulten K, Voth GA (2004) The mechanism of proton exclusion in aquaporin channels. Proteins 55:223-228

Jensen MØ, Mouritsen OG (2006) Single-channel water permeabilities of Escherichia coli aquaporins AqpZ and GlpF. Biophys J 90:2270-2284

Jensen MØ, Tajkhorshid E, Schulten K (2001) The mechanism of glycerol conduction in aquaglyceroporins. Structure 9:1083-1093

Jensen MØ, Park S, Tajkhorshid E, Schulten K (2002) Energetics of glycerol conduction through aquaglyceroporin GlpF. Proc Natl Acad Sci USA 99:6731-6736

Jensen MØ, Tajkhorshid E, Schulten K (2003) Electrostatic tuning of permeation and selectivity in aquaporin water channels. Biophys J 85:2884-2899

Jung JS, Preston GM, Smith BL, Guggino WB, Agre P (1994) Molecular structure of the water channel through aquaporin CHIP - the hourglass model. J Biol Chem 269:14648-14654

Kato M, Pisliakov AV, Warshel A (2006) The barrier for proton transport in aquaporins as a challenge for electrostatic models: the role of protein relaxation in mutational calculations. Proteins 64:829-844

Lee JK, Kozono D, Remis J, Kitagawa Y, Agre P, Stroud RM (2005) Structural basis for conductance by the archaeal aquaporin AqpM at 1.68 A. Proc Natl Acad Sci USA 102:18932-18937

Maurel C, Reizer J, Schroeder JI, Chrispeels MJ, Saier MH (1994) Functional characterization of the Eschericia coli glycerol facilitator, GlpF, in Xenopus oocytes. J Biol Chem 269: $11869-11872$

Murata K, Mitsuoka K, Walz T, Agre P, Heymann JB, Engel A, Fujiyoshi Y (2000) Structural determinants of water permeation through aquaporin-1. Nature 407:599-605

Nakhoul NL, Davis BA, Romero MF, Boron WF (1998) Effect of expressing the water channel aquaporin-1 on the $\mathrm{CO}_{2}$ permeability of Xenopus oocytes. Am J Physiol Cell Physiol 274:C543-C548

Prasad GVR, Coury LA, Finn F, Zeidel ML (1998) Reconstituted aquaporin 1 water channels transport $\mathrm{CO}_{2}$ across membranes. J Biol Chem 273:33123-33126

Preston GM, Carroll TP, Guggino WB, Agre P (1992) Appearance of water channels in Xenopus oocytes expressing red-cell CHIP28 protein. Science 256:385-387

Savage DF, Egea PF, Robles-Colmenares Y, O'Connell JDI, Stroud RM (2003) Architecture and

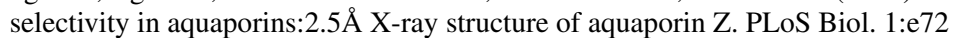

Sui H, Han B-G, Lee JK, Walian P, Jap BK (2001) Structural basis of water-specific transport through the AQP1 water channel. Nature 414:872-878

Tajkhorshid E, Nollert P, Jensen MØ, Miercke LJW, O’Connell J, Stroud RM, Schulten K (2002) Control of the selectivity of the aquaporin water channel family by global orientational tuning. Science 296:525-530

Törnroth-Horsefield S, Wang Y, Hedfalk K, Johanson U, Karlsson M, Tajkhorshid E, Neutze R, Kjellbom P (2006) Structural mechanism of plant aquaporin gating. Nature 439:688-694

Torrie GM, Valleau JP (1974) Monte Carlo free energy estimates using non-Boltzmann sampling: application to the sub-critical Lennard-Jones fluid. Chem Phys Lett 28:578-581

Uehlein N, Lovisolo C, Siefritz F, Kaldenhoff R (2003) The tobacco aquaporin NtAQP1 is a membrane $\mathrm{CO}_{2}$ pore with physiological functions. Nature 425:734-737

Wang Y, Schulten K, Tajkhorshid E (2005) What makes an aquaporin a glycerol channel? A comparative study of AqpZ and GlpF. Structure 13:1107-1118

Wang Y, Cohen J, Boron WF, Schulten K, Tajkhorshid E (2007) Exploring gas permeability of cellular membranes and membrane channels with molecular dynamics. J Struct Biol 157: 534-544

Yang B, Fukuda N, van Hoek A, Matthay MA, Ma T, Verkman AS (2000) Carbon dioxide permeability of aquaporin-1 measured in erythrocytes and lung of aquaporin-1 null mice and in reconstituted proteoliposomes. J Biol Chem 275:2686-2692

Zardoya R (2005) Phylogeny and evolution of the major intrinsic protein family. Biol Cell 97: 397-414 
Zeidel ML, Ambudkar SV, Smith BL, Agre P (1992) Reconstitution of functional water channels in liposomes containing purified red-cell CHIP28 protein. Biochemistry 31:7436-7440

Zeidel ML, Nielsen S, Smith BL, Ambudkar SV, Maunsbach AB, Agre P (1994) Ultrastructure, pharmacological inhibition, and transport selectivity of aquaporin channel-forming integral protein in proteoliposomes. Biochemistry 33:1606-1615

Zhu F, Tajkhorshid E, Schulten K (2002) Pressure-induced water transport in membrane channels studied by molecular dynamics. Biophys J 83:154-160

Zhu F, Tajkhorshid E, Schulten K (2004a) Collective diffusion model for water permeation through microscopic channels. Phys Rev Lett 93:224501

Zhu F, Tajkhorshid E, Schulten K (2004b) Theory and simulation of water permeation in aquaporin-1. Biophys J 86:50-57 